\title{
Amplitude analyses with charm decays at LHCb
}

\author{
Alberto Corrêa dos Reis* \\ Centro Brasileiro de Pesquisas Físicas. \\ E-mail: alberto@cbpf.br
}

Studies of the $K \pi$ and $K \bar{K}$ S-wave amplitudes are presented using the Cabibbo-suppressed $D^{0} \rightarrow$ $K_{S}^{0} K^{ \pm} \pi^{\mp}$ and the doubly Cabibbo-suppressed $D^{+} \rightarrow K^{-} K^{+} K^{+}$decays. The data correspond, respectively, to 3 and $2 \mathrm{fb}^{-1}$ of $p p$ collisions collected by the LHCb experiment. A comparison between the $(K \pi)^{0}$ and $(K \pi)^{ \pm}$S-wave amplitudes is made from the Dalitz plot analysis of the $D^{0} \rightarrow K_{S}^{0} K^{ \pm} \pi^{\mp}$ decays. The $K \bar{K}$ S-wave amplitude is obtained from the Dalitz plot analysis of the $D^{+} \rightarrow K^{-} K^{+} K^{+}$decay, performed for the first time for this channel. All amplitudes are determined using the isobar model.

VIII International Workshop On Charm Physics

5-9 September, 2016

Bologna, Italy

* Speaker.

${ }^{\dagger}$ on behalf of the LHCb collaboration. 


\section{Introduction}

Over fifty years have passed since the birth of the Quark Model and yet the identification of the $I\left(J^{P}\right)=0\left(0^{+}\right)$states remains unclear. Thanks to the richness of QCD, different forms of bound states of quarks and gluons are allowed: glueballs, hybrids, molecules, and tetraquarks. Some of these "exotic" states could share the same quantum numbers and populate the same region of the spectrum (up to $2 \mathrm{GeV}$ ) as the usual $q \bar{q}$ scalar mesons.

Unfortunately, there is no process in which the scalars can be studied in a model-independent way. The primary source of information on low energy hadron-hadron interactions are the reactions $\pi N \rightarrow \pi \pi N$ and $K N \rightarrow K \pi N[1,2,3]$. The $\pi \pi \rightarrow \pi \pi$ and $K \pi \rightarrow K \pi$ scattering amplitudes are determined in these reactions selecting events with low momentum transfer, which restricts the analyses to a kinematic region where the contribution from one-pion-exchange amplitude is expected to be dominant. The $\pi \pi$ and $K \pi$ scattering is actually the interaction between the incident pion or kaon with a virtual "pion", close to the mass shell. Other contributions such as the $\rho$-exchange are neglected. In the case of the $K \bar{K} \rightarrow K \bar{K}$, nosuch data exist.

The understanding of the the scalar mesons requires the combination of information from different processes: central production, $p \bar{p}$ annihilation, kaon and $J / \psi$ decays and, more recently, decays of $D$ and $B$ mesons. The latter are indeed an interesting alternative, not only because scalar mesons are abundantly produced, but also because the $\pi \pi, K \pi$ and $K \bar{K}$ spectrum can be accessed continuosly, starting from threshold and covering the whole elastic region.

In recent years the $D^{+} \rightarrow K^{-} \pi^{+} \pi^{+}[4,5,6]$ and $D^{+} \rightarrow \pi^{-} \pi^{+} \pi^{+}$[7] decays have been used to study the $K \pi$ and $\pi \pi \mathrm{S}$-wave amplitudes using an approach that has the least model dependence. The interpretation of these results, however, is not straightforward. Nonleptonic decays of a heavy meson can be seen as a two-stage process. The weak transition of the heavy quark and the hadron formation, which is often referred to as the weak vertex, is followed by the strong interaction between the final state hadrons (FSI). There is no clear separation between these two stages. FSI involving all hadrons may add slowly varying phases across the phase space $[8,9]$. Slowly varying phases can also be inherited from the weak part of the decay. The S-wave phases need to be disentangled from these effects.

In this note, model-dependent Dalitz plot analyses of the Cabibbo-suppressed decays $D^{0} \rightarrow$ $K_{S}^{0} K^{ \pm} \pi^{\mp}$ [10] and the doubly Cabibbo-suppressed decay $D^{+} \rightarrow K^{-} K^{+} K^{+}$[11] are used to investigate the $K \pi$ and $K \bar{K}$ S-wave amplitudes. The decays $D^{0} \rightarrow K_{S}^{0} K^{ \pm} \pi^{\mp}$ offer an unique opportunity to study both the charged and neutral $K \pi$ systems in a single process. The Dalitz plot analysis of the $D^{+} \rightarrow K^{-} K^{+} K^{+}$decay is performed for the first time for this channel, and is focused in the identification of the key features of the $K \bar{K} \mathrm{~S}$-wave amplitude. All results presented in here are obtained using the isobar model [12].

\section{Formalism}

The formalism for the Dalitz plot analysis of the $D^{0} \rightarrow K_{S}^{0} K^{ \pm} \pi^{\mp}$ and $D^{+} \rightarrow K^{-} K^{+} K^{+}$decays is very standard. The distribution of events across the Dalitz plot, represented in terms of the two invariants $s_{12} \equiv\left(p_{1}+p_{2}\right)^{2}$ and $s_{13} \equiv\left(p_{1}+p_{3}\right)^{2}$, is given by 


$$
\frac{d \Gamma}{d s_{12} d s_{13}}=\frac{1}{(2 \pi)^{3} 32 M_{D}^{3}}\left|\mathscr{M}\left(s_{12}, s_{13}\right)\right|^{2},
$$

where $M_{D}$ is the $D^{+}$mass and $p_{i}$ are the four-momenta of the final state particles. The Lorentz invariant amplitude, $\mathscr{M}$, is described by a coherent sum of a nonresonant amplitude, assumed to be constant over the phase space, and resonant amplitudes,

$$
\mathscr{M}\left(s_{12}, s_{13}\right)=\sum c_{n} A_{n}\left(s_{12}, s_{13}\right) .
$$

For a resonance in the $s 12$ system, the resonant amplitudes $A_{k}$ are defined as

$$
A_{n}\left(s_{12}, s_{13}\right)=F_{D} \times F_{R} \times M_{n}\left(s_{12}\right) \times \mathscr{S}\left(s_{12}, s_{13}\right)
$$

where the form factors $F_{D}$ and $F_{R}$, for the $D^{+}$and the resonance decays, respectively, are parameterized by the Blatt-Weisskopf barrier factors [13], the spin amplitudes, $\mathscr{S}$, accounting for the conservation of angular momentum, are derived using the covariant tensor formalism [10], and the dynamical function, $M_{n}$, describing the respective two-body lineshape, is a Breit-Wigner function in most cases, except where mentioned. The complex coefficients $c_{n}$ are the fit parameters.

The optimum values of the fit parameters in the decay amplitude $\mathscr{M}$ are determined by an unbinned maximum likelihood fit. The likelihood function is formed combining the signal and background PDFs,

$$
\mathscr{L}=\prod_{\text {events }}\left\{f_{S}^{i} \times S_{\mathrm{PDF}}\left(s_{12}^{i}, s_{13}^{i}\right)+\left(1-f_{S}^{i}\right) \times B_{\mathrm{PDF}}\left(s_{12}^{i}, s_{13}^{i}\right)\right\},
$$

where $f_{S}^{i}$ is the signal fraction, which depends on the three-body invariant mass of the $i$-th event.

The results are expressed in terms of the magnitude, phase and fit fraction for each channel. The fit fractions are defined in a convention-independent way, calculated by integrating the squared modulus of the corresponding amplitude over the phase space, and dividing by the integral of the total amplitude squared

$$
\mathrm{FF}_{n}=\frac{\int\left|c_{n} A_{n}\left(s_{12}, s_{13}\right)\right|^{2} \mathrm{~d} s_{12} \mathrm{~d} s_{13}}{\int\left|\sum_{i} c_{i} A_{i}\left(s_{12}, s_{13}\right)\right|^{2} \mathrm{~d} s_{12} \mathrm{~d} s_{13}}
$$

The sum of fit fractions for all channels is in general different from $100 \%$ due to the presence of interferences. The interference fit fractions between the resonances $i$ and $j(i<j)$ are defined by

$$
\mathrm{FF}_{i j}=\frac{\int 2 \operatorname{Re}\left[c_{i} c_{j}^{*} A_{i} A_{j}^{*}\right] \mathrm{d} s_{12} \mathrm{~d} s_{13}}{\int\left|\sum_{n} c_{n} A_{n}\left(s_{12}, s_{13}\right)\right|^{2} \mathrm{~d} s_{12} \mathrm{~d} s_{13}} .
$$

To access the goodness-of-fit, the Dalitz plots are divided into bins of variable size. In each bin a $\chi^{2}$ is calculated from the observed and predicted population. A p-value is obtained from the sum of the $\chi^{2}$ values from all bins.

\section{Study of the $D^{0} \rightarrow K_{S}^{0} K^{ \pm} \pi^{\mp}$ decays}

The $D^{0} \rightarrow K_{S}^{0} K^{ \pm} \pi^{\mp}$ decays are interesting for a variety of reasons. When produced through $B^{-} \rightarrow D^{0} K^{-}$, these decay modes can be used to measure the CKM angle $\gamma$. They could also be 
used in time-dependent studies for the determination of charm mixing parameters and $\mathrm{CP}$ violation searches. Here the focus is on the study of the $(K \pi)^{0, \pm} \mathrm{S}$-wave amplitude. The analysis is performed using the full LHCb Run1 data set, corresponding to $3 \mathrm{fb}^{-1}$ of $p p$ collisions at $\sqrt{s}=7$ and $8 \mathrm{TeV}$.

The flavour of the $D^{0}$ is determined by the charge of the slow pion, $\pi_{s}^{+}$, in the decay chain $D^{*+} \rightarrow D^{0} \pi_{s}^{+}$. The branching fraction of the $D^{0} \rightarrow K_{S}^{0} K^{-} \pi^{+}$mode is approximately 1.7 times larger than that of the $D^{0} \rightarrow K_{S}^{0} K^{+} \pi^{-}$channel [14]. The signal yields are determined from twodimensional fits to the $m\left(K_{S}^{0} K \pi\right)$ and $\Delta m \equiv m\left(K_{S}^{0} K \pi \pi_{s}^{+}\right)-m\left(K_{S}^{0} K \pi\right)$ distributions. The signals

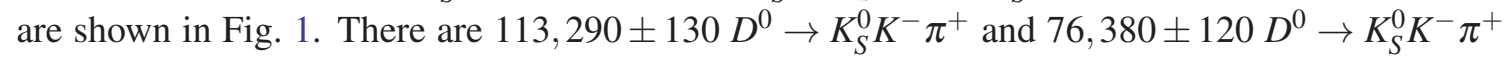
candidates.
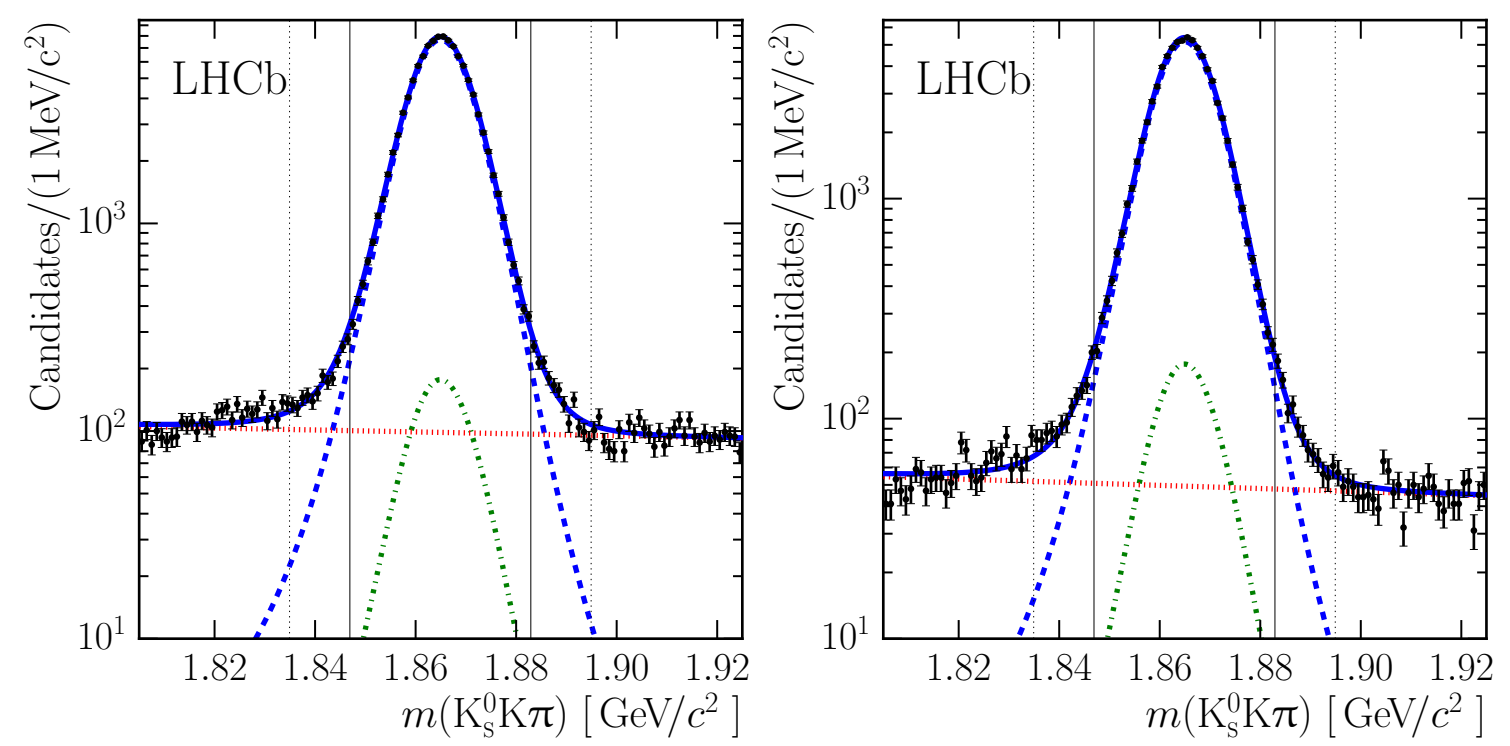

Figure 1: Mass distributions for the $D^{0} \rightarrow K_{S}^{0} K^{-} \pi^{+}$(left) and $D^{0} \rightarrow K_{S}^{0} K^{+} \pi^{-}$(right), with fit result superimposed. The dotted red curve represents the combinatorial background, whereas the dash-dotted green curve represents the contribution from real $D^{0}$ mesons combined with incorrect $\pi_{s}^{+}$.

The Dalitz plot analysis is performed using events in the regions delimited by the vertical solid lines in Fig. 1. The distribution of these events in the the Dalitz plot is shown in Fig. 2.

Two models for the $K \pi \mathrm{S}$-wave amplitudes are tested. They replace the usual sum of BreitWigner amplitudes and are referred to as LASS and GLASS. These forms include both the $K_{0}^{*}(1430)$ resonance and a nonresonant component. The LASS parameterization takes the form

$$
A_{0}=f(x) \frac{m_{K \pi}}{q} \sin \left(\delta_{S}+\delta_{F}\right) e^{i\left(\delta_{S}+\delta_{F}\right)}
$$

where $f(x)=A \exp \left(b_{1} x+b_{2} x^{2}+b_{3} x^{3}\right)\left(x \equiv m_{K \pi} / m_{K_{0}^{*}(1430)}\right)$ is an empirical real production form factor and $q$ is the momentum of the kaon or pion in the $K \pi$ rest frame. The phases are defined by

$$
\tan \delta F=\frac{2 a q}{2+a r q^{2}}, \quad \tan \delta_{S}=\frac{m_{R} \Gamma\left(m_{K \pi}\right)}{m_{R}^{2}-m_{K \pi}^{2}}
$$



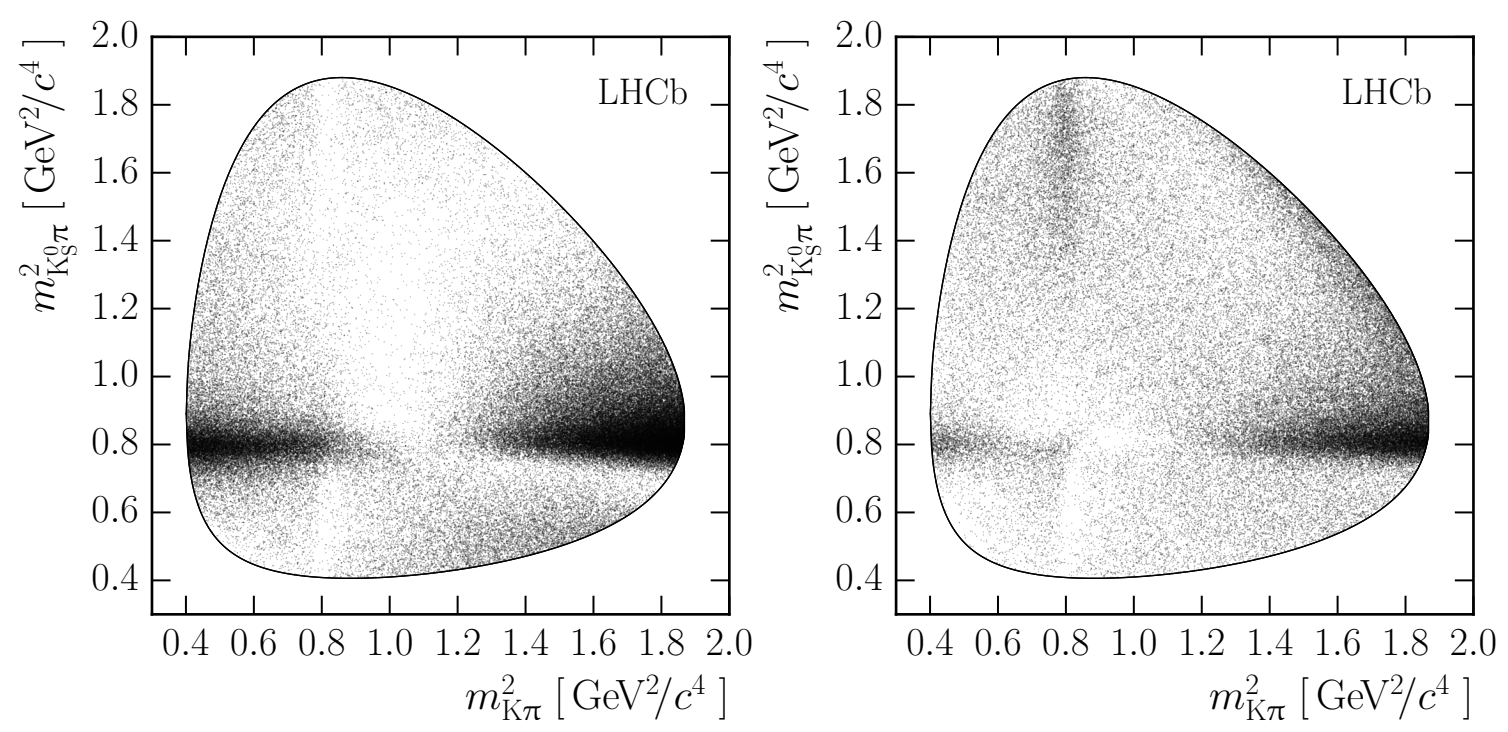

Figure 2: The Dalitz plots of the $D^{0} \rightarrow K_{S}^{0} K^{-} \pi^{+}$(left) and $D^{0} \rightarrow K_{S}^{0} K^{+} \pi^{-}$(right) candidates in the signal region.

The values of the scattering length $a$, effective range $r$ and the $K_{0}^{*}(1430)$ mass, $m_{R}$ are taken from the LASS experiment [3]. The function $\Gamma\left(m_{K \pi}\right)$ represents the mass-dependent width of the $K_{0}^{*}(1430)$.

The GLASS parameterization introduces new free parameters which imply some freedom with respect to the phase from the LASS model. In addition, in the fits with the GLASS model the phases of the charged and neutral $K \pi \mathrm{S}$-wave amplitudes may be different. This additional freedom may accommodate effects arising from the different production mechansms of the charged and neutral $K \pi$ systems. The GLASS amplitude is defined by

$$
A_{0}=\left[F \sin \left(\delta_{F}+\phi_{F}\right) e^{i\left(\delta_{F}+\phi_{F}\right)}+\sin \left(\delta_{S}\right) e^{i\left(\delta_{S}+\phi_{S}\right)} e^{2 i\left(\delta_{F}+\phi_{F}\right)}\right] \frac{m_{K \pi}}{q},
$$

where $\delta_{F}$ and $\delta_{S}$ are defined as before, and $F, \phi_{F}$ and $\phi_{S}$ are free parameters in the fit.

In the $D^{0} \rightarrow K_{S}^{0} K^{ \pm} \pi^{\mp}$ there are many possible resonances in all two-body systems. It is impractical to have all of them in a single fit model. Decay models with different combinations were tested. Only those with moderate interference fit fractions were kept. Resonances are included if the increase in $-2 \log \mathscr{L}$ is larger than 25 units.

In the Dalitz plot fit all parameters except the complex coefficients $c_{n}$ are shared between the PDFs for the two decay modes. The parameters defining the LASS S-wave are shared between the charged and neutral $K \pi$ system, whereas in the GLASS fit they are allowed to differ. Fits with equivalent quality are obtained with both $K \pi \mathrm{S}$-wave parameterizations.

The fit fractions of the dominant contributions in the best isobar models are summarized in Tables 1 and 2 for $D^{0} \rightarrow K_{S}^{0} K^{-} \pi^{+}$and $D^{0} \rightarrow K_{S}^{0} K^{+} \pi^{-}$, respectively. In both decay modes, the dominant contribution is the $K^{*}(892)^{ \pm}$amplitude, followed by the sum of the $K \pi$ S-wave amplitudes. The uncertainties on the fit fraction of the $K \pi \mathrm{S}$-waves are large, especially in the GLASS fit. In both modes a very large fraction for the $K^{*}(1410)$ amplitude is observed. This is surpris- 


\begin{tabular}{lcc}
\hline Resonance & LASS & GLASS \\
\hline$K^{*}(892)^{+}$ & $56.9 \pm 0.6 \pm 1.1$ & $57.0 \pm 0.8 \pm 2.6$ \\
$K^{*}(1410)^{+}$ & $9.6 \pm 1.1 \pm 2.9$ & $5 \pm 1 \pm 4$ \\
$\left(K_{S}^{0} \pi\right)_{S-\text { wave }}^{+}$ & $11.7 \pm 1.0 \pm 2.3$ & $12 \pm 2 \pm 9$ \\
& & \\
$K^{*}(892)^{0}$ & $2.5 \pm 0.2 \pm 0.2$ & $2.5 \pm 0.25 \pm 0.4$ \\
$\bar{K}^{*}(1410)^{0}$ & $3.8 \pm 0.5 \pm 2.0$ & $9 \pm 1 \pm 4$ \\
$\left(K_{S}^{0} \pi\right)_{S-\text { wave }}^{0}$ & $18 \pm 2 \pm 4$ & $11 \pm 2 \pm 10$ \\
& & \\
$a_{0}(980)^{-}$ & $4.0 \pm 0.7 \pm 1.1$ & - \\
\hline
\end{tabular}

Table 1: Fit fractions (\%) of the dominant contributions to the $D^{0} \rightarrow K_{S}^{0} K^{-} \pi^{+}$Dalitz plot, according to the best isobar models using LASS and GLASS parameterizations of the $K \pi$ S-wave amplitude.

\begin{tabular}{lcc}
\hline Resonance & LASS & GLASS \\
\hline$K^{*}(892)^{-}$ & $28.8 \pm 0.4 \pm 1.3$ & $29.5 \pm 0.6 \pm 1.6$ \\
$K^{*}(1410)^{-}$ & $11.9 \pm 1.5 \pm 2.2$ & $3.1 \pm 0.6 \pm 1.6$ \\
$\left(K_{S}^{0} \pi\right)_{S-\text { wave }}^{-}$ & $6.3 \pm 0.9 \pm 2.1$ & $5.4 \pm 0.9 \pm 1.7$ \\
$K^{*}(892)^{0}$ & $4.8 \pm 0.2 \pm 0.4$ & $5.2 \pm 0.2 \pm 0.3$ \\
$\bar{K}^{*}(1410)^{0}$ & $2.2 \pm 0.6 \pm 2.1$ & $9 \pm 1 \pm 4$ \\
$\left(K_{S}^{0} \pi\right)_{S-\text { wave }}^{0}$ & $17 \pm 2 \pm 6$ & $12 \pm 1 \pm 8$ \\
& & \\
$a_{0}(980)^{+}$ & $26 \pm 2 \pm 10$ & $11 \pm 1 \pm 6$ \\
\hline
\end{tabular}

Table 2: Fit fractions (\%) of the dominant contributions to the $D^{0} \rightarrow K_{S}^{0} K^{+} \pi^{-}$Dalitz plot, according to the best isobar models using LASS and GLASS parameterizations of the $K \pi \mathrm{S}$-wave amplitude.

ing given the small coupling of this resonance to $K \pi(6.6 \%)$ and the limited phase space of the $D^{0} \rightarrow K^{*}(1410)^{ \pm} K^{\mp}$ decay. In the $D^{0} \rightarrow K_{S}^{0} K^{+} \pi^{-}$decay a large contribution from the $K_{S}^{0} K^{+}$ $\mathrm{S}$-wave is observed, although the uncertainty on the fit fraction is large.

A comparison between the S-wave phase variation of the LASS and GLASS paramaterizations is shown in Fig. 3. A good description of the data is obtained with a single phase common to both charged and neutral $K \pi$ systems, as in the LASS model. However, an equally good description is given by the GLASS model, where the phases not only deviate from that of LASS, but are also different for $(K \pi)^{0}$ and $(K \pi)^{ \pm}$. Moreover, the GLASS phases are very different from those obtained by the BaBar collaboration, with the same formulation, in the $D^{0} \rightarrow K_{S}^{0} \pi^{-} \pi^{+}$decay. This difference may result from FSI involving all final-state particles, or from the difference between the topologies of the weak vertices. In the $D^{0} \rightarrow K_{S}^{0} K^{\mp} \pi^{p m}$ and $D^{0} \rightarrow K_{S}^{0} \pi^{+} \pi$ - decays there are Swave amplitudes in all two-body subsystems. The interference between the $\mathrm{S}$-waves in each decay mode may also explain the differences observed in Fig. 3. 


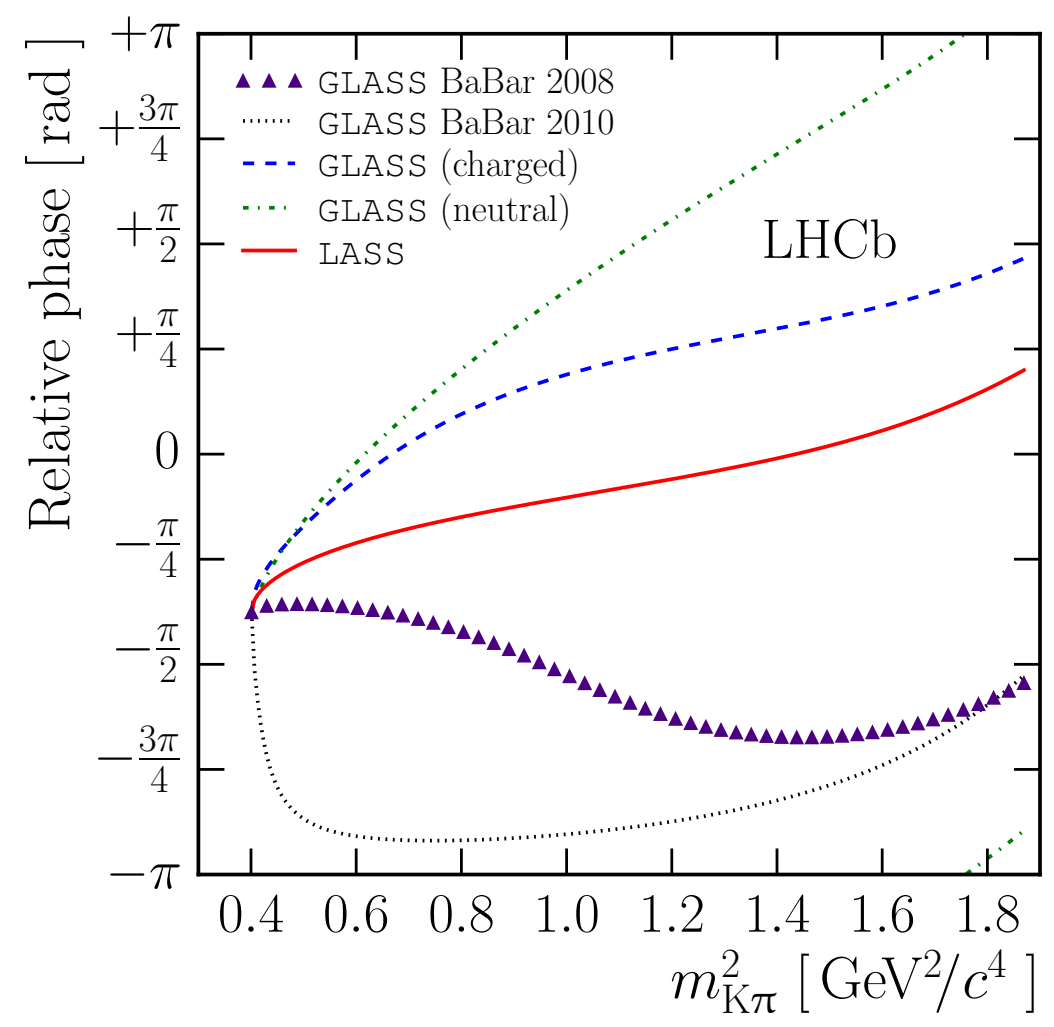

Figure 3: Comparison of the $K \pi \mathrm{S}$-wave phase variation of the various parameterizations used. The solid red curve represents the phase from the LASS parametrization, while the dashed blue and dash-dotted green curves show the GLASS phase for the charged and neutral $K \pi$ systems, respectively. The final two curves show the GLASS forms fitted to the charged $K \pi$ S-wave from the $D^{0} \rightarrow K_{S}^{0} \pi^{+} \pi^{-}$decays in Ref. [15] (triangular markers, purple) and Ref. [16] (dotted curve, black).

\section{Study of the $D^{+} \rightarrow K^{-} K^{+} K^{+}$decay}

The main purpose of the Dalitz plot analysis of the $D^{+} \rightarrow K^{-} K^{+} K^{+}$decay is to identify the key features of the $K^{+} K^{-} \mathrm{S}$-wave amplitude. The data set used in the analysis corresponds to 2 $\mathrm{fb}^{-1}$ of $p p$ collisions at $\sqrt{s}=8 \mathrm{TeV}$, collected in 2012 by the LHCb experiment. This is the first Dalitz plot analysis of this mode.

The mass plot of selected events is shown in Fig. 4. The sample contains approximately 100,000 fully reconstructed decays with $90 \%$ purity. In the Dalitz plot fit only events in the $m\left(K^{-} K^{+} K^{+}\right)$interval $[1861.42,1879.48] \mathrm{MeV} / c^{2}$ are used.

The Dalitz plot of the $D^{+} \rightarrow K^{-} K^{+} K^{+}$decays from the signal region is shown in Fig. 5, after background subtraction and efficiency correction. A concentration of events at the $\phi(1020)$ mass squared is the only prominent structure. The remainder of the events are evenly distributed across the Dalitz plot. The $\phi$ is a vector resonance. Conservation of angular momentum gives rise to an angular distribution of the decay products that gives to the $\phi$ band a " $U$ " shape when seen from the crossed channel. The asymmetry between the two lobes of the $\phi$ is caused by the interference between the P-and S-waves. The latter populates the whole phase space. 

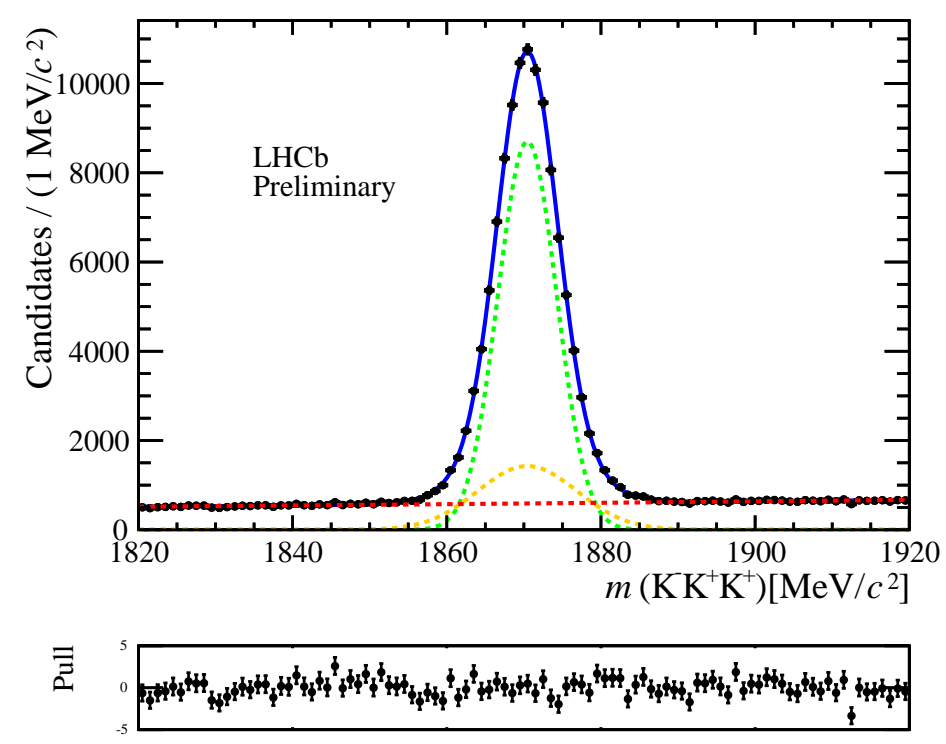

Figure 4: The $K^{-} K^{+} K^{+}$mass spectrum of selected candidates (data points), with the fit result overlaid (solid blue line). The green and yellow dashed lines indicate the two Gaussian functions representing the signal. The red line represents the background.
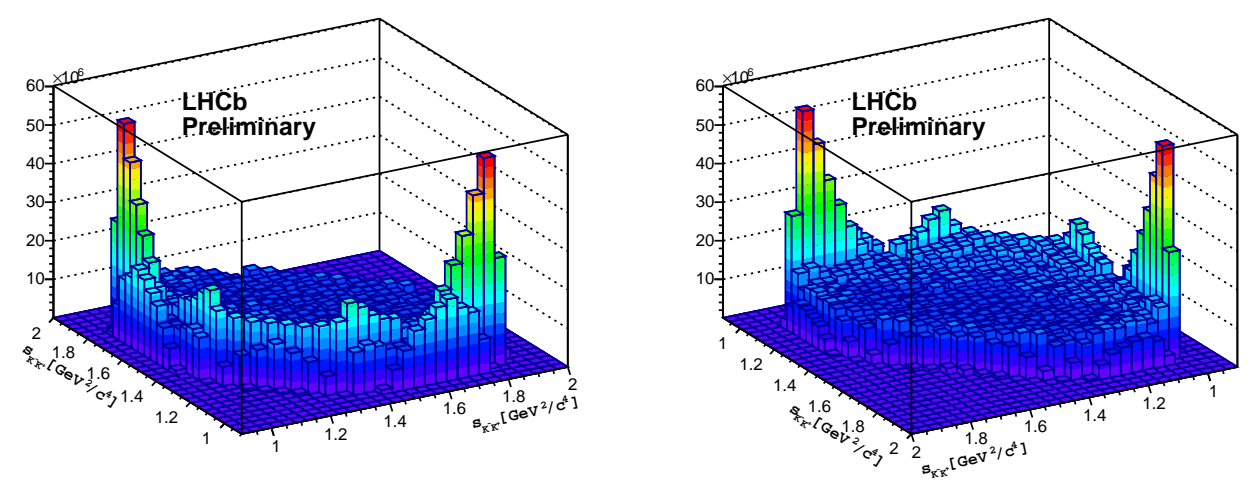

Figure 5: Two 3D views of the background subtracted and efficiency corrected $D^{+} \rightarrow K^{-} K^{+} K^{+}$Dalitz plot.

There are two tree-level amplitudes for the $D^{+} \rightarrow K^{-} K^{+} K^{+}$, namely the $c \bar{d} \rightarrow u \bar{s}$ (annihilation) and the $c \rightarrow d u \bar{s}$ (W-radiation). In the latter, a $d \bar{d}$ source hadronizes into a $K^{0} \bar{K}^{0}$ pair, which turns into a $K^{-} K^{+}$pair by rescattering. It is difficult to estimate the relative strenght of these amplitudes, but the system coming out of the $c \bar{d}$ annihilation has no isospin constraint. The scalar component of the $K^{-} K^{+} K^{+}$final state can, therefore, contain resonances from both the $f_{0}\left(I^{P}=0^{+}\right)$and $a_{0}$ $\left(I^{P}=1^{+}\right)$families. In particular, the states that may contribute are the $f_{0}(980), f_{0}(1370)$ and $f_{0}(1500)$, and the $a_{0}(980)$ and $a_{0}(1450)$. There could be also a contribution from a nonresonant amplitude, which is usually incorporated in the S-wave. Given the small phase space of this decay, spin-2 resonances are not expected to have a significant contribution.

In practice it is very difficult to disentangle the $f_{0}(980)$ from the $a_{0}(980)$, since the nominal 
mass of these states lie below the kinematic limit (the $K^{-} K^{+}$threshold) of the Dalitz plot. The same happens in the other end of the $K^{-} K^{+}$spectrum, where it is difficult to separate the $a_{0}(1450)$, $f_{0}(1370)$ and $f_{0}(1500)$.

Three combinations of resonances are tested. All combinations include, in addition to the $\phi$ resonance, a state close to the $K^{-} K^{+}$threshold and a broad amplitude at higher $K^{-} K^{+}$mass. The resonance at low $K^{-} K^{+}$mass is always the $f_{0}(980)$. For the higher part of the $K^{-} K^{+}$spectrum three possibilities are considered: a constant nonresonant amplitude (Model 1), the $a_{0}(1450)$ (Model 2) and the $f_{0}(1370)$ (Model 3). Except for the $f_{0}(980)$, which is represented by a Flatté function [17], all resonances are described by relativistic Breit-Wigner functions. For the $f_{0}(1370)$, a still controversial state with large uncertainty on its line shape, the Breit-Wigner mass and width are free parameters.

Model 1 fails to describe the data. A significant improvement on the fit quality is obtained replacing the real constant amplitude by a Breit-Wigner function, as in Models 2 and 3. This is an indication that a phase variation compatible with that of a resonance is necessary at higher values of $m\left(K^{-} K^{+}\right)$. The fit results for Models 2 and 3 are summarized in Table 3.

Table 3: Dalitz plot fit results for Models 2 and 3. For each entry, the first uncertainty is due to statistics and the second due to systematics.

\begin{tabular}{|c|c|c|c|}
\hline Model 2 & & & \\
\hline Channel & magnitude & phase $\left[{ }^{\circ}\right]$ & fit fraction $(\%)$ \\
\hline$f_{0}(980) K^{+}$ & $3.20 \pm 0.12 \pm 0.13$ & $-59.3 \pm 5.1 \pm 4.1$ & $26.5 \pm 1.5 \pm 1.7$ \\
\hline$f_{0}(X) K^{+}$ & $3.52 \pm 0.58 \pm 0.48$ & $13.8 \pm 8.1 \pm 6.5$ & $22.1 \pm 3.2 \pm 2.6$ \\
\hline$\phi(1020) K^{+}$ & $1[\mathrm{fix}]$ & $0[f i x]$ & $6.5 \pm 0.1 \pm 0.1$ \\
\hline \multicolumn{4}{|l|}{ Model 3} \\
\hline Channel & magnitude & phase $\left[{ }^{\circ}\right]$ & fit fraction $(\%)$ \\
\hline$f_{0}(980) K^{+}$ & $3.40 \pm 0.06 \pm 0.06$ & $-71.8 \pm 1.3 \pm 0.9$ & $29.9 \pm 1.0 \pm 0.7$ \\
\hline$a_{0}(1450) K^{+}$ & $3.42 \pm 0.06 \pm 0.04$ & $44.6 \pm 3.4 \pm 1.6$ & $18.6 \pm 0.6 \pm 0.5$ \\
\hline$\phi(1020) K^{+}$ & $1[\mathrm{fix}]$ & $0[f i x]$ & $6.5 \pm 0.1 \pm 0.1$ \\
\hline
\end{tabular}

Fits with similar quality are obtained using Models 2 and 3. In both fits the S-wave is the dominant contribution. The fit fraction of the $\phi$ amplitude is the same in both models. The sum of fit fractions is approximately 55\% due to a large interference between the two components of the $\mathrm{S}$-wave. The $\phi$ amplitude has little interference with the S-wave, which limits the possibility of determining the S-wave phase using the techinque developed by the E791 collaboration [4].

A comparison between the magnitude and the phase motion of the S-wave, as given by the three models, is shown in Fig. 6. The magnitude and phase from Models 2 and 3 are very similar, and they differ significantly from those of Model 1.

\section{Discussion}

Amplitude analysis of multi-body, nonleptonic decays of $D$ and $B$ mesons have been used to study the light meson spectrum, with emphasis on the scalar sector. Information from heavy flavour 

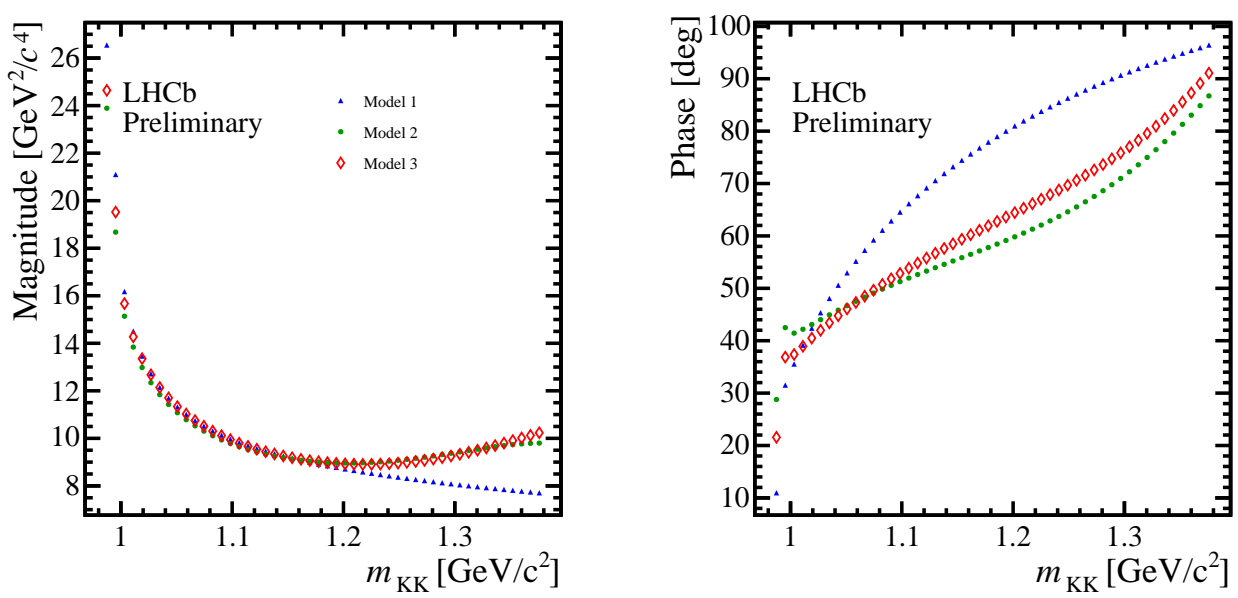

Figure 6: The magnitude and phase variation of the $S$-wave amplitude, as a function of the $K^{-} K^{+}$invariant mass, for the three models tested.

decays is complementary to those from other reactions, such as $\pi N, K N$ scattering, $p \bar{p}$ annihilation, central production and $J / \psi$ decays.

The analysis of the $D^{0} \rightarrow K_{S}^{0} \pi^{ \pm}, K^{ \pm} \pi^{\mp}$ decays allows the simultaneous study of the S-wave amplitude of both neutral and charged $K \pi$ systems. The Dalitz plot of both decay modes can be described by a single amplitude for $(K \pi)^{0}$ and $(K \pi)^{ \pm}$with the phase variation from the LASS experiment. An equally good description of the data is possible using the GLASS model, where the phases of the $(K \pi)^{0}$ and $(K \pi)^{ \pm}$S-wave amplitude are allowed to differ. In this case, the two phases deviate significantly from each other and from that of the LASS model. The interpretation of these results is not straightforward given the interplay between the three possible S-wave amplitudes in the $D^{0} \rightarrow K_{S}^{0} \pi^{ \pm}, K^{ \pm} \pi^{\mp}$ decays. This study will benefit from mode data and more sophisticated analysis tools.

The resonant structure of the $D^{+} \rightarrow K^{-} K^{+} K^{+}$decay is investigated for the first time. The S-wave amplitude is the dominant contribution. Similar dominance is also observed in other three-body final states with a pair of identical particles, such as $D^{+} \rightarrow K^{-} \pi^{+} \pi^{+}$and $D_{(s)}^{+} \rightarrow$ $\pi^{-} \pi^{+} \pi^{+}[14]$.

At higher $K^{-} K^{+}$mass, the data is best described by models in which the variation of the Swave phase is consistent to that of a resonance. Such a phase variation is also necessary at the lower part of the $K^{-} K^{+}$spectrum. Limitations of the isobar model, however, prevent the identification of the states forming the $K^{-} K^{+} \mathrm{S}$-wave. At each end of the $K^{-} K^{+}$spectrum the line shapes of the possible candidates are very similar, since all resonances have large widths and masses that lie very close - either in or out - to the kinematic limit. At low mass, the two possible contributions are the $f_{0}(980)$ and the $a_{0}(980)$. At the upper part of the spectrum, the possible states are the $f_{0}(1370)$, $a_{0}(1450)$ and the $f_{0}(1500)$. With more data and more sophysticate tools, a model-independent determination of the $K^{-} K^{+}$S-wave phase may be possible.

With the increasing size of the data sets, the development of better analysis tools is a crucial task. In the case of the $D^{+} \rightarrow K^{-} K^{+} K^{+}$decay, a model based on unitarized chiral perturbation 
theory [18] is being tested on data. In this model, the relative contribution of individual components is fixed by the theory.

\section{References}

[1] Hyams, B. et al. [CERN-Munich collaboration] $\pi \pi$ Phase Shift Analysis from $600 \mathrm{MeV}$ to $1900 \mathrm{MeV}$ Nucl. Phys. B64 (1973) 134

[2] Grayer, G. et al. [CERN-Munich collaboration] High Statistics Study of the Reaction $\pi^{-} p \rightarrow \pi^{-} \pi^{+} n$ : Apparatus, Method of Analysis, and General Features of Results at $17 \mathrm{GeV} / \mathrm{c}$ Nucl. Phys. $\mathbf{B} 75$ (1974) 189

[3] Aston, D. et al. [LASS collaboration], A Study of $K^{-} \pi^{+}$Scattering in the Reaction $K^{-} \pi^{+} \rightarrow K^{-} \pi^{+} n$ at $11 \mathrm{GeV} / \mathrm{c}$ Nucl. Phys. B296 (1988) 493

[4] Aitala, E. M. et al. [E791 collaboration], Model-independent measurement of $S$-wave $K^{-} \pi^{+}$systems using $D^{+} \rightarrow K^{-} \pi^{+} \pi^{+}$decays from Fermilab E791, Phys. Rev. $D 73$ (2006) 059901.

[5] Bonvicini, G. et al. [CLEO collaboration], Dalitz plot analysis of the $D^{+} \rightarrow K^{-} \pi^{+} \pi^{+}$decay, Phys. Rev. $D 78$ (2008), 052001

[6] Link, J. . M. et al. [FOCUS collaboration], The $K^{-} \pi^{+} S$-wave from the $D^{+} \rightarrow K^{-} \pi^{+} \pi^{+}$decay, Phys. Lett B681 (2009) 14.

[7] Aubert, B. et al. [BaBar collaboration], Dalitz plot analysis of $D_{s}^{+} \rightarrow \pi^{-} \pi^{+} \pi^{+}$Phys. Rev. D 79 (2009) 032003

[8] Magalhães, P. C. et al. Towards three-body unitarity in $D^{+} \rightarrow K^{-} \pi^{+} \pi^{+}$Phys. Rev. D 84 (2011) 094001

[9] Guimarães, K. S. F. F. et al. Final state interaction in $D^{+} \rightarrow K^{-} \pi^{+} \pi^{+}$with $K \pi I=1 / 2$ and $3 / 2$ channels JHEP 08 (2014) 135

[10] Aaij, R. et al. [LHCb collaboration], Studies of the resonance structure in $D^{0} \rightarrow K_{S}^{0} K^{ \pm} \pi^{\mp}$ decays Phys. Rev. D 93 (2016) 052018

[11] Aaij, R. et al. [LHCb collaboration], Dalitz plot analysis of the $D^{+} \rightarrow K^{-} K^{+} K^{+}$decay with the isobar model LHCb-CONF-2016-008 (2016)

[12] Nakamura, K. et al. [Particle Data Group], Review of Particle Physics, Dalitz plot analysis formalism J. Phys. G 37 (2010) 075021

[13] Blatt, J. and Weisskopf, J., Theoretical Nuclear Physics, John Wiley and Sons, 1952.

[14] Patrignani, C. et al. [Particle Data Group], Chin. Phys. C 40 (2016), 100001

[15] Aubert, B. et al. [BaBar collaboration], Improved measurement of the CKM angle $\gamma$ in $B^{\mp} \rightarrow D^{(*)} K^{(*) \mp}$ decays with a Dalitz plot analysis of $D$ decays to $K_{S}^{0} \pi^{-} \pi^{+}$and $K_{S}^{0} K^{-} K^{+}$Phys. Rev. $D 78$ (2008) 034023

[16] P. del Amo Sanchez et al. [BaBar collaboration], Measurement of $D^{0}-\bar{D}^{0}$ mixing parameters using $K_{S}^{0} \pi^{-} \pi^{+}$and $K_{S}^{0} K^{-} K^{+}$decays Phys. Rev. Lett. 105 (2010) 081803

[17] Flatté, S. M., Coupled-channel analysis of the $\pi \eta$ and $K \bar{K}$ systems near $K \bar{K}$ threshold, Phys. Lett. B63 (1976) 224

[18] R.T. Aoude, P.C. Magalhães, A. C. dos Reis and M.R. Robilotta, Multi-Meson Model applied to $D^{+} \rightarrow K^{-} K^{+} K^{+}$, [arXiv:1604.02904]. See also P. Magalhães contribution to these proceedings. 